Open Access

\title{
Decoupled beamforming techniques for distributed MIMO two-way multi-relay networks with imperfect CSI
}

\author{
Wei Duan ${ }^{1,2}$, Xiaojun Zhu ${ }^{1,4}$, Li Jin ${ }^{1}$, Wei Wang ${ }^{1,4}$, Guoan Zhang ${ }^{1 *}$ and Jeaho Choi ${ }^{3}$
}

\begin{abstract}
This paper investigates decoupled beamforming techniques for the distributed multi-input multi-output (MIMO) two-way relay networks (TWRN) with imperfect channel state informations (CSIs). The objective of this paper is to maximize the weighted sum rate (SR) with semi-infinite relay power constraints. Since the objective problem is difficult to be solved directly, considering the high signal-to-residual-interference-plus-noise ratio (SRINR) and employing the Cauchy-Schwarz inequality and S-lemma, the problem can be approximately converted into a source beamforming decoupled one. In addition, with the optimal relay beamforming design and maximum ratio combining (MRC) at the receiver, the MIMO channels are decoupled into parallel single-input single-output (SISO) channels. By this way, the suboptimal relay beamforming matrix can be efficiently obtained with minimal relay power constraint. Specifically, the semi-infinite constraints can be reformulated into a linear matrix inequality (LMI), which can be efficiently solved by using an alternating optimization algorithm. Numerical results demonstrate that our proposed decoupled beamforming scheme outperforms the existing works in terms of the SR and the computational complexity with satisfactory convergence.
\end{abstract}

Keywords: Two-way relay network, MIMO, Amplify-and-forward, Beamforming design, LMI, Imperfect CSI

\section{Introduction}

Recently, cooperative and multiple-input multiple-output (MIMO) systems [1-4] have been widely considered as a candidate for the fifth generation (5G) wireless communication due to their transmission reliability, where multiple users (MU) are simultaneously served at the same frequency band by the base station (BS) equipped with number of antennas. Cooperative two-way relay network (TWRN) technology has attracted significant interests due to the superior spectral efficiency. Several relaying schemes haven proposed, i.e., amplify-and-forward (AF) [5-8], decode-and-forward (DF) [9, 10], and denoiseand-forward (DNF) [11, 12]. Specifically, the cooperative relaying beamforming design has been studied in [13-16], where the authors showed the optimal solutions of the source and/or relay beamforming matrices. Moreover, the spatially correlated fading channels are

*Correspondence: gzhang@ntu.edu.cn

${ }^{1}$ School of Electronics and Information, Nantong University, Nantong, China

Full list of author information is available at the end of the article considered in [17, 18], which is a more practical assumption but makes the training problem more challenging. The authors of [17] presented training designs for estimation of spatially correlated MIMO AF two-way multi-relay channels, where an optimal training structure is initially derived to minimize total mean-square-error (MSE) of the channel estimation. Based on [17], an optimal training scheme is efficiently designed to minimize the total MSE of the channel estimation under the transmit power constraints at the source nodes and at the relay in [18]. In addition, the authors considered a TWRN with an AF protocol over either two, three, or four time slots [19].

Considering inaccurate channel estimation and feedback delay, the perfect channel state information (CSI), which is proposed in the above works [5-18], is usually hard to obtain in practice. By taking account into the channel uncertainties, the imperfect CSI scenario has been studied in [20-25]. In [20], joint relay and jammer selection and power control for physical layer security issues in two-way relay networks are studied to maximize 
the secrecy capacity of the network. In [21], the authors addressed the robust multiple-antenna relay design problem in TWRN and provided the robust multiple-antenna relay design based on the channel estimates. A low complexity processing matrices are presented for a multi-pair two-way massive MIMO AF full-duplex relay system [22]. Specifically, in [23], the problem of optimal beamforming and power allocation for an AF-based TWRN is studied in the presence of interference and CSI uncertainty, where two different approaches, namely the total power minimization method and the signal-to-interference-plusnoise-ratio (SINR) balancing technique, are proposed. Particularly, the robust two-way relay precoder design for a cognitive radio network is investigated in [24], where two different types of CSI errors with corresponding robust designs are proposed. It is worth noting, in [25], the joint optimal robust beamforming designs in multipair two-way nonregenerative relaying systems, where an insightful closed form solution is obtained that is not only robust to the imperfect CSI but also adjustable to various CSI circumstances at the users and the relay.

However, most existing works for the robust two-way relaying networks were unavoidable to focus on the joint design of source and relay beamforming matrices that may lead to a higher computational complexity. In addition, as shown in [26], for the multiple relays scheme, the performance of the capacity outperforms that of the single relay one. On the other hand, multiple relays scheme is more practical and challenging for the wireless communication scenarios. Moreover, the weighed scheme can be regarded as one kind of the resource allocations, which is general. Therefore, in order to reduce the complexity, our study aims to design a decoupled beamforming scheme for the distributed MIMO two-way relaying scheme with imperfect and reciprocal CSI. By converting the weighted sumrate (SR) maximization problem into a source beamforming decoupled one whose target is to maximize the signal-to-residual-interference-plus-noise ratio (SRINR) by means of approximations, the S-lemma, and the Cauchy-Schwarz inequality, the objective problem can be efficiently solved by an alternating algorithm. With the decoupled source beamforming design and the maximum ratio combining (MRC) at the receiver, the optimal relay beamforming can be efficiently obtained which leads to that the MIMO channels can be decoupled into parallel single-input single-output (SISO) channels. Numerical results are presented to corroborate that the performance of the decoupled scheme is improved and to compare it to the existing works with satisfactory convergence.

The rest of this paper is organized as follows. Section 2 describes the system model of the TWRN and objective problems. In Section 3, the proposed decoupled beamforming and optimal relay beamforming designs are investigated. Numerical results are presented to show the excellent performance of our proposed scheme in Section 4. Section 5 concludes this paper.

Notations: For an $M \times N$ matrix $\mathbf{A}, \mathrm{E}(\mathbf{A}),\|\mathbf{A}\|, \mathbf{A}^{T}$, $\operatorname{tr}(\mathbf{A})$, vec(A), and $\mathbf{A}^{\dagger}$ denote the statistical expectation, Frobenius norm, transpose, trace, vectorization, and Hermitian transpose of A, respectively. $\mathbf{I}_{N}$ represents an $N \times N$ identity matrix.

\section{System model and optimal relay beamforming design}

\subsection{System model}

Consider a simple two-way relay channels (TWRC) consisting of two user equipments, i.e., $\mathrm{UE}_{1}$ and $\mathrm{UE}_{2}$, and $L$ relay nodes $\left\{R_{1}, R_{2}, \ldots, R_{L}\right\}$ as shown in Fig. 1 . Each source and relay nodes are equipped with $M$ and $N$ antennas, respectively. Assume that all nodes operate in half-duplex mode and the direct link between two UEs does not exist. By taking the estimation error into account, the CSI is assumed to be reciprocal and partially known at each node. Denote the estimated channels from the $\mathrm{UE}_{t}$ to the relay $R_{i}$, from the relay $R_{i}$ to the $\mathrm{UE}_{t}$ by $\widetilde{\mathbf{F}}_{i, t} \in \mathbb{C}^{N \times M}$ and $\widetilde{\mathbf{G}}_{t, i} \in \mathbb{C}^{M \times N}$, for $t \in\{1,2\}$, respectively, as follows:

$$
\mathbf{F}_{i, t} \triangleq\left\{\widetilde{\mathbf{F}}_{i, t}+\Delta_{\mathbf{F}_{i, t}}\right\}
$$

and

$$
\mathbf{G}_{t, i} \triangleq\left\{\widetilde{\mathbf{G}}_{t, i}+\Delta_{\mathbf{G}_{t, i}}\right\}
$$

with $\left\{\Delta_{\mathbf{F}_{i, t}}, \Delta_{\mathbf{G}_{t, i}}\right\}$ as the channel uncertainties. For a better readability and distinction, in this paper, we simply consider two channel matrices $\left(\mathbf{F}_{i, t}, \mathbf{G}_{t, i}\right)$ with $\mathbf{F}_{i, t}=\mathbf{G}_{t, i}^{T}$ and $\mathbf{F}_{i, \bar{t}}=\mathbf{G}_{\bar{t}, i}^{T}$. Since that, the channel uncertainty is provided by the inaccurate channel estimation, feedback delay, and so on. Therefore, the norm bounded error (NBE) of the channel uncertainty is normally considered as a small slack value [27], which is a reasonable assumption in a practical system. Therefore, in this paper, the channel uncertainties are considered as a NBE model for its simplicity:

$$
\left\|\Delta_{\mathbf{F}_{i, 1}}\right\|=\left\|\Delta_{\mathbf{G}_{1, i}}\right\|=\alpha_{i},\left\|\Delta_{\mathbf{F}_{i, 2}}\right\|=\left\|\Delta_{\mathbf{G}_{2, i}}\right\|=\beta_{i},
$$

where $0 \leq\left\{\alpha_{i}, \beta_{i}\right\} \ll 1$. By this way, a worst-case design methodology can be adopted, resulting in the proposed system design which maximizes performance for the worst possible CSI realization as defined by the NBE.

It is assumed that two UEs exchange the message using two consecutive time slots. At the first time slot, the information data $\mathbf{x}_{t}=\left[x_{1, t}, x_{2, t}, \ldots, x_{m, t}\right]^{T}$, with $\mathrm{E}\left(\mathbf{x}_{t} \mathbf{x}_{t}^{\dagger}\right)=$ $\mathbf{I}_{M}$, for $i \in\{1, \ldots, M\}$, is linearly processed by a precoding matrix $\mathbf{B}_{t} \in \mathbb{C}^{M \times M}$ with the power constraint 


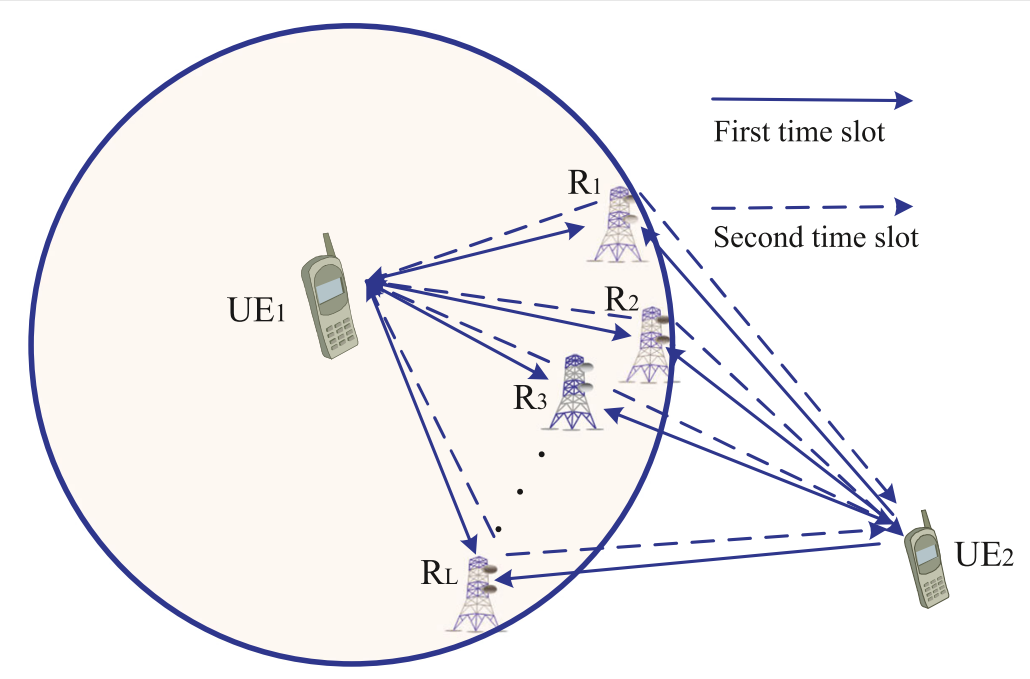

Fig. 1 Two-way MIMO multi-relay networks

$\left\|\mathbf{B}_{t}\right\|^{2} \leq P_{t}$ and then transmitted to the relay nodes. Thus, the received signal at $R_{i}$ can be expressed as

$$
\mathbf{y}_{R_{i}}=\mathbf{F}_{i, 1} \mathbf{B}_{1} \mathbf{x}_{1}+\mathbf{F}_{i, 2} \mathbf{B}_{2} \mathbf{x}_{2}+\mathbf{n}_{R_{i}},
$$

where $\mathbf{n}_{R_{i}} \sim \mathcal{C N}\left(0, \sigma_{R_{i}}^{2} \mathbf{I}_{N}\right)$ denotes the additive white Gaussian noise (AWGN) vector with zero mean and variance $\sigma_{R_{i}}^{2} \mathbf{I}_{N}$.

At the second time slot, the relay node $R_{i}$ generates the transmit signal $\mathbf{W}_{i} \mathbf{y}_{R_{i}}$, for $\left\|\mathbf{W}_{i}\right\|^{2} \leq \omega_{i}$, with the $N \times N$ beamforming matrix $\mathbf{W}_{i}$ and forwards to the $\mathrm{UE}_{1}$ and $\mathrm{UE}_{2}$ which results in $\mathbf{x}_{R_{i}}=\mathbf{W}_{i} \mathbf{y}_{R_{i}}$ with the average transmit power as

$$
\mathrm{E}\left\{\left\|\mathbf{x}_{R_{i}}\right\|_{2}^{2}\right\}=\left\|\mathbf{W}_{i} \mathbf{F}_{i, 1} \mathbf{B}_{1}\right\|^{2}+\left\|\mathbf{W}_{i} \mathbf{F}_{i, 2} \mathbf{B}_{2}\right\|^{2}+\sigma_{R_{i}}^{2}\left\|\mathbf{W}_{i}\right\|^{2},
$$

where we have assumed that the elements of the data stream vector $\mathbf{x}_{t}$ transmitted by each user are independent, i.e., $\mathbf{E}\left\{\mathbf{x}_{t} \mathbf{x}_{t}^{\dagger}\right\}=\mathbf{I}_{M}$. Thus, the received signal at source node $S_{t}$, for $t \in\{1,2\}$, becomes

$$
\mathbf{y}_{t}=\sum_{i=1}^{L} \mathbf{G}_{t, i} \mathbf{W}_{i} \mathbf{y}_{R_{i}}+\mathbf{n}_{t},
$$

where $\mathbf{n}_{t}$ denotes the noise vector at the source node $S_{t}$ with zero mean and variance $\sigma_{S_{t}}^{2} \mathbf{I}_{N}$.

In practical systems, however, achieving perfect SIC is a hard problem due to channel estimation errors. Thus, the self-interference cannot be completely eliminated which leads to the residual self-interference cannot be ignorable. In order to subside the adverse effect of the interference, with the estimated channel coefficients, the final obser- vation of the residual self-interference at the $\mathrm{UE}_{\bar{t}}$ can be obtained as

$$
\begin{aligned}
\chi_{\bar{t}} & =\sum_{i=1}^{L}\left(\mathbf{G}_{\bar{t}, i} \mathbf{W}_{i} \mathbf{F}_{i, \bar{t}} \mathbf{B}_{\bar{t}} \mathbf{x}_{\bar{t}}-\widetilde{\mathbf{G}}_{\bar{t}, i} \mathbf{W}_{i} \widetilde{\mathbf{F}}_{i, \bar{t}} \mathbf{B}_{\bar{t}} \mathbf{x}_{\bar{t}}\right) \\
& =\sum_{i=1}^{L}(\widetilde{\mathbf{G}}_{\bar{t}, i} \mathbf{W}_{i} \Delta_{\mathbf{F}_{i, \bar{t}}}+\Delta_{\mathbf{G}_{\bar{t}, i}} \mathbf{W}_{i} \widetilde{\mathbf{F}}_{i, \bar{t}}+\underbrace{\Delta_{\mathbf{G}_{\bar{t}, i}} \mathbf{W}_{i} \Delta_{\mathbf{F}_{i, \bar{t}}}}_{\mathcal{K}}) \mathbf{B}_{\bar{t}} \mathbf{x}_{\bar{t}} .
\end{aligned}
$$

It is clear that the covariance of the term $\mathcal{K}$ can be obtained as $\left\|\Delta_{\mathbf{G}_{\bar{t}, i}} \mathbf{W}_{i} \Delta_{\mathbf{F}_{i, t}}\right\|^{2}$. Since that, the norm bounded errors of $\left\{\Delta_{\mathbf{G}_{\bar{t}, i}}, \Delta_{\mathbf{F}_{i, \bar{t}}}\right\}$ are assumed to be small slack values, which is a reasonable assumption in a practical system, i.e., $0 \leq\left\{\alpha_{i}, \beta_{i}\right\} \ll 1$. Using $\|\mathbf{A B}\| \leq\|\mathbf{A}\|\|\mathbf{B}\|$, we have $\left\|\Delta_{\mathbf{G}_{\bar{t}, i}} \mathbf{W}_{i} \Delta_{\mathbf{F}_{i, \bar{t}}}\right\|^{2} \leq\left\{\alpha_{i}^{4}\left\|\mathbf{W}_{i}\right\|^{2}, \beta_{i}^{4}\left\|\mathbf{W}_{i}\right\|^{2}\right\}$, which is very close to 0 . Therefore, in this paper, the negligible term involving only CSI uncertainty is omitted, which is practical and relatively easy to achieve.

Since the sum of the individual rate in a MIMO system is difficult to be obtained, therefore, we turn to design the relay beamforming to recast the optimization problem into the one of achievable data rate for each data stream.

\subsection{Optimal relay beamforming design}

Suppose the singular value decomposition (SVD) of $\widetilde{\mathbf{G}}_{\bar{t}, i}$ and $\widetilde{\mathbf{F}}_{i, t}$ as follows

$$
\widetilde{\mathbf{G}}_{\bar{t}, i}=\Pi_{\bar{t}, i}\left[\left[\mathbf{G}_{\bar{t}, i}^{\diamond}\right]_{M \times M} \mathbf{0}_{M \times(N-M)}\right] \mathbf{V}_{\bar{t}, i}^{\dagger} \triangleq \Pi_{\bar{t}, i} \Sigma_{\bar{t}, i} \mathbf{V}_{\bar{t}, i}^{\dagger}
$$


and

$$
\widetilde{\mathbf{F}}_{i, t}=\mathbf{U}_{i, t}\left[\begin{array}{c}
{\left[\mathbf{F}_{i, t}^{\diamond}\right]_{M \times M}} \\
\mathbf{0}_{(N-M) \times M}
\end{array}\right] \Omega_{i, t}^{\dagger} \triangleq \mathbf{U}_{i, t} \Gamma_{i, t} \Omega_{i, t}^{\dagger},
$$

where $\mathbf{V}_{\bar{t}, i} \in \mathbb{C}^{N \times N}, \mathbf{U}_{i, t} \in \mathbb{C}^{N \times N}, \Pi_{\bar{t}, i} \in \mathbb{C}^{M \times M}$, and $\Omega_{i, t} \in \mathbb{C}^{M \times M}$ are unitary matrices as well as $\Sigma_{\bar{t}, i}=$ $\left[\left[\mathbf{G}_{\bar{t}, i}^{\diamond}\right]_{M \times M} \mathbf{0}_{M \times(N-M)}\right]$ and $\Gamma_{i, t}=\left[\begin{array}{l}{\left[\mathbf{F}_{i, t}^{\diamond}\right]_{M \times M}} \\ \mathbf{0}_{(N-M) \times M}\end{array}\right]$.

Theorem 1 Using the SVDs in (7) and (8), the optimal relay beamforming matrix as the solution to the objective problem $T_{3}$ can be obtained as

$$
\mathbf{W}_{i}=\mathbf{V}_{\bar{t}, i} \mathbf{C}_{i} \mathbf{U}_{i, t}^{\dagger},
$$

where $\mathbf{C}_{i} \in \mathbb{C}^{N \times N}$ is a matrix to be determined.

Proof The proof is similar to $[8,28]$.

Without loss of generality, $\mathbf{C}_{i}$ can be further partitioned as follows:

$$
\mathbf{C}_{i}=\left[\begin{array}{cc}
{\left[\mathbf{W}_{i}^{\diamond}\right]_{M \times M}} & \mathbf{X}_{M \times(N-M)} \\
\mathbf{Y}_{(N-M) \times M} & \mathbf{Z}_{(N-M) \times(N-M)}
\end{array}\right] .
$$

On the one hand, since the channels from different relays, the estimated channel, and its corresponding channel uncertainties are independent, denoting $\tilde{\chi}_{\bar{t}}=$ $\sum_{i=1}^{L}\left(\widetilde{\mathbf{G}}_{\bar{t}, i} \mathbf{W}_{i} \Delta_{\mathbf{F}_{i, \bar{t}}}+\Delta_{\mathbf{G}_{\bar{t}, i}} \mathbf{W}_{i} \widetilde{\mathbf{F}}_{i, \bar{t}}\right)$, we have

$$
\begin{aligned}
\left\|\tilde{\chi}_{\bar{t}}\right\|^{2} \leq & \sum_{i=1}^{L} \varrho_{\bar{t}, i}^{2}\left(\left\|\mathbf{G}_{\bar{t}, i}^{\diamond}\right\|^{2}+\left\|\mathbf{F}_{i, \bar{t}}^{\diamond}\right\|^{2}\right) \\
& \times\left\|\left[\begin{array}{ll}
{\left[\mathbf{W}_{i}^{\diamond}\right]_{M \times M}} & \mathbf{X}_{M \times(N-M)} \\
\mathbf{Y}_{(N-M) \times M} & \mathbf{Z}_{(N-M) \times(N-M)}
\end{array}\right]\right\|^{2},
\end{aligned}
$$

where $\varrho_{t, i}=\alpha_{i}$ for $t=1$ as long as $\varrho_{t, i}=\beta_{i}$ for $t=2$. On the other hand, since the channel uncertainty $\Delta_{\mathbf{F}_{i, t}}$ can be further partitioned following

$$
\Delta_{\mathbf{F}_{i, t}}=\left[\begin{array}{c}
{\left[\Delta_{\mathbf{F}_{i, t}}^{\diamond}\right]_{M \times M}} \\
{\left[\Delta_{\mathbf{F}_{i, t}^{b}}^{b}\right]_{(N-M) \times M}}
\end{array}\right],
$$

upon substituting (9), (10), and (12) into the relay power constraint (4), we have

$$
\begin{aligned}
& \mathbf{E}\left\{\left\|\mathbf{x}_{R_{i}}\right\|_{2}^{2}\right\} \\
& =\operatorname{tr}\left\{\mathbf{W}_{i} \mathbf{y}_{R_{i}} \mathbf{y}_{R_{i}}^{\dagger} \mathbf{W}_{i}^{\dagger}\right\} \\
& =\operatorname{tr}\left\{\mathbf{W}_{i}\left(\mathbf{F}_{i, 1} \mathbf{B}_{1} \mathbf{B}_{1}^{\dagger} \mathbf{F}_{i, 1}^{\dagger}+\mathbf{F}_{i, 2} \mathbf{B}_{2} \mathbf{B}_{2}^{\dagger} \mathbf{F}_{i, 2}^{\dagger}+\sigma_{R_{i}}^{2} \mathbf{I}_{N}\right) \mathbf{W}_{i}^{\dagger}\right\} \\
& =\operatorname{tr}\left\{\mathbf{W}_{i}^{\diamond}\left(\sum_{t=1}^{2} \mathbf{F}_{i, t}^{\sharp} \mathbf{B}_{t}\left(\mathbf{F}_{i, t}^{\sharp} \mathbf{B}_{t}\right)^{\dagger}+\sigma_{R_{i}}^{2} \mathbf{I}_{M}\right)\left(\mathbf{W}_{i}^{\diamond}\right)^{\dagger}+\mathbf{X} \Phi_{i} \mathbf{X}^{\dagger}\right\} \\
& \quad+\operatorname{tr}\left\{\mathbf{Z} \Phi_{i} \mathbf{Z}^{\dagger}+\mathbf{Y}\left(\sum_{t=1}^{2} \mathbf{F}_{i, t}^{\sharp} \mathbf{B}_{t}\left(\mathbf{F}_{i, t}^{\sharp} \mathbf{B}_{t}\right)^{\dagger}+\sigma_{R_{i}}^{2} \mathbf{I}_{M}\right) \mathbf{Y}^{\dagger}\right\},
\end{aligned}
$$

where $\mathbf{F}_{i, t}^{\sharp}=\mathbf{F}_{i, t}^{\diamond}+\Delta_{\mathbf{F}_{i, t}}^{\diamond}$ and $\Phi_{i}=\sum_{t=1}^{2} \Delta_{\mathbf{F}_{i, t}}^{b} \mathbf{B}_{t}\left(\Delta_{\mathbf{F}_{i, t}}^{b} \mathbf{B}_{t}\right)^{\dagger}+$ $\sigma_{R_{i}}^{2} \mathbf{I}_{N-M}$. From (11) and (13), it is clear that, for any feasible $\mathbf{C}_{i}$ with $\{\mathbf{X}, \mathbf{Y}, \mathbf{Z}\} \neq \mathbf{0}$, one can always find

$$
\mathbf{C}_{i}^{\prime}=\left[\begin{array}{cc}
{\left[\mathbf{w}_{i}^{\diamond}\right.} & \mathbf{0}_{M \times M \times(N-M)} \\
\mathbf{0}_{(N-M) \times M} & \mathbf{0}_{(N-M) \times(N-M)}
\end{array}\right],
$$

which can achieve the smaller relay and interference power constraints. By this way, the optimal expression of $\mathbf{W}_{i}$ with minimal relay and interference power constraints can be represented as

$$
\mathbf{W}_{i}^{\sharp}=\mathbf{V}_{\bar{t}, i}\left[\begin{array}{cc}
{\left[\mathbf{W}_{i}^{\diamond}\right.} & \mathbf{0}_{M \times M \times(N-M)} \\
\mathbf{0}_{(N-M) \times M} & \mathbf{0}_{(N-M) \times(N-M)}
\end{array}\right] \mathbf{U}_{i, t}^{\dagger} .
$$

\subsection{Objective problem}

It is easy to see that the actual equivalent channel from $\mathrm{UE}_{t}$ to $\mathrm{UE}_{\bar{t}}$ is with dimension $M \times M$. Therefore, by employing the $\mathrm{MRC}$ at $\mathrm{UE}_{\bar{t}}$, the equivalent received signals from each channel can be linearly added together. With the condition $\mathrm{E}\left(\mathbf{x}_{t} \mathbf{x}_{t}^{\dagger}\right)=\mathbf{I}_{M}$, the objective weighted sum-rate (SR) problem for the total system can be recast into the problem achievable data rate for each data stream, i.e., the MIMO channels are decoupled into $M$ parallel SISO channels. With these observations, from (4), (6), and (15) similar to [29-31], the received SRINR over the $m$ th channel $(1 \leq m \leq M)$ for $\mathrm{UE}_{\bar{t}}$ can be simply written as

$$
\zeta_{\bar{t}}^{(m)}=\frac{\left\|\sum_{i=1}^{L} \mathbf{G}_{\bar{t}, i} \mathbf{W}_{i} \mathbf{F}_{i, t} \mathbf{B}_{t}\right\|^{2}}{\left\|\tilde{\chi}_{\bar{t}} \mathbf{B}_{\bar{t}}\right\|^{2}+\sigma_{R_{i}}^{2}\left\|\sum_{i=1}^{L} \mathbf{G}_{\bar{t}, i} \mathbf{W}_{i}\right\|^{2}+\sigma_{S_{t}}^{2}},
$$

where $\bar{t}=2$ for $t=1$ while $\bar{t}=1$ for $t=2$. Based on which, we have the weighted SR maximization problem as

$$
\begin{aligned}
T_{1}: \max _{\mathbf{B}_{t}, \mathbf{W}_{i}} & \sum_{t=1}^{2} \lambda_{\bar{t}} \log \left(1+\frac{\left\|\mathbf{H}_{t} \mathbf{B}_{t}\right\|^{2}}{\left\|\widetilde{\chi}_{\bar{t}} \mathbf{B}_{\bar{t}}\right\|^{2}+N_{t}}\right) \\
\text { s.t. } & (4) \leq P_{R_{i}}, \quad\left\|\mathbf{B}_{t}\right\|^{2} \leq P_{t}, \forall t=\{1,2\},
\end{aligned}
$$


where $\mathbf{H}_{t}=\sum_{i=1}^{L} \mathbf{G}_{\bar{t}, i} \mathbf{W}_{i} \mathbf{F}_{i, t}, N_{t}=\sigma_{R_{i}}^{2}\left\|\sum_{i=1}^{L} \mathbf{G}_{\bar{t}, i} \mathbf{W}_{i}\right\|^{2}+$ $\sigma_{S_{t}}^{2}$, and $P_{R_{i}}$ is the maximum allocated power to the relay. In addition, the weight term $\lambda_{t}$ is determined depending on the required quality of service (QoS).

\section{Decoupled source beamforming designs}

Since the problem $T_{1}$ is neither convex nor concave and the optimal $\mathbf{B}_{t}$ is intractable, it is difficult to obtain the globally optimal solution. In this section, we propose a decoupled way to obtain the sub-optimal solution of the worst-case weighted SR.

Considering the high signal-to-interference-plus-noise ratio (SINR), the objective weighted SR $R_{\text {sum }}$ in (17) can be approximately reexpressed as

$$
\begin{aligned}
R_{\text {sum }} & \approx \lambda_{1} \log \frac{\left\|\mathbf{H}_{2} \mathbf{B}_{2}\right\|^{2}}{\left\|\tilde{\chi}_{1} \mathbf{B}_{1}\right\|^{2}+N_{1}}+\lambda_{2} \log \frac{\left\|\mathbf{H}_{1} \mathbf{B}_{1}\right\|^{2}}{\left\|\widetilde{\chi}_{2} \mathbf{B}_{2}\right\|^{2}+N_{2}} \\
& =\log \frac{\left(\left\|\mathbf{H}_{2} \mathbf{B}_{2}\right\|^{2}\right)^{\lambda_{1}}}{\left(\left\|\tilde{\chi}_{2} \mathbf{B}_{2}\right\|^{2}+N_{2}\right)^{\lambda_{2}}}+\log \frac{\left(\left\|\mathbf{H}_{1} \mathbf{B}_{1}\right\|^{2}\right)^{\lambda_{2}}}{\left(\left\|\widetilde{\chi}_{1} \mathbf{B}_{1}\right\|^{2}+N_{1}\right)^{\lambda_{1}}} .
\end{aligned}
$$

It is interesting to see that, since $\log (\cdot)$ is a monotonic function and assuming the nominal CSI and the channel errors are independent, with fixed $\lambda_{t}$, from (31), the objective problem $T_{1}$ can be equivalently converted into the following problem as

$$
\begin{aligned}
T_{2}: & \max _{\mathbf{B}_{t}, \mathbf{W}_{i}} \prod_{t=1}^{2} \gamma_{t} \text { for } t=1,2, \\
\text { s.t. } & q_{t}\left(\mathbf{B}_{t}\right) \geq \gamma_{t},(4) \leq P_{R_{i}},\left\|\mathbf{B}_{t}\right\|^{2} \leq P_{t},
\end{aligned}
$$

where $q_{t}\left(\mathbf{B}_{t}\right)=\frac{\left(\left\|\mathbf{H}_{t} \mathbf{B}_{t}\right\|^{2}\right)^{\lambda_{\bar{t}}}}{\left(\left\|\widetilde{\chi}_{t} \mathbf{B}_{t}\right\|^{2}+N_{t}\right)^{\lambda_{t}}}$ and $\gamma_{t}$ is a slack value, where, similar to $[29,32-34]$, by introducing a slack value $a$, the optimization problem $\{\max \mathcal{A}\}$ can be equivalently rewrite as $\{\max a\}$ s.t. $\mathcal{A} \geq a$. In order to relax $N_{t}$ in $q_{t}\left(\mathbf{B}_{t}\right)$, after introducing the slack value $s_{t}$, we have

$$
\sum_{i=1}^{L}\left(\varrho_{t, i}^{2}+\left\|\widetilde{\mathbf{G}}_{\bar{t}, i} \mathbf{W}_{i}\right\|^{2}\right) \geq \frac{\varsigma_{t}-\sigma_{1}^{2}}{\sigma_{R_{i}}^{2}}
$$

which leads to $N_{t} \geq \varsigma_{t}$, where $\varrho_{t, i}=\alpha_{i}$ for $t=1$ as long as $\varrho_{t, i}=\beta_{i}$ for $t=2$, and $\tau_{t}$ is a slack value. With this obser- vation and employing the Cauchy-Schwarz inequality, we have

$$
\begin{aligned}
\frac{\left(\left\|\mathbf{H}_{t} \mathbf{B}_{t}\right\|^{2}\right)^{\lambda_{\bar{t}}}}{\left(\left\|\tilde{\chi}_{t} \mathbf{B}_{t}\right\|^{2}+N_{t}\right)^{\lambda_{t}}} & =\frac{\operatorname{tr}\left(\mathbf{B}_{t}^{\dagger} \mathbf{H}_{t}^{\dagger} \mathbf{H}_{t} \mathbf{B}_{t}\right)^{\lambda_{\bar{t}}}}{\operatorname{tr}\left[\mathbf{B}_{t}^{\dagger}\left(\tilde{\chi}_{t}^{\dagger} \tilde{\chi}_{t}+\frac{\zeta t}{m P_{t}} \mathbf{I}_{M}\right) \mathbf{B}_{t}\right]^{\lambda_{t}}} \\
& \leq \frac{\operatorname{tr}\left(\mathbf{H}_{t}^{\dagger} \mathbf{H}_{t}\right)^{\lambda_{\bar{t}}}}{\operatorname{tr}\left[\left(\tilde{\chi}_{t}^{\dagger} \tilde{\chi}_{t}+\frac{\zeta t}{m P_{t}} \mathbf{I}_{M}\right)\right]^{\lambda_{t}} P_{t}^{2\left(\lambda_{t}-\lambda_{\bar{t}}\right)}} \\
& =\frac{\left(\left\|\mathbf{H}_{t}\right\|^{2}\right)^{\lambda_{\bar{t}}}}{\left(\left\|\tilde{\chi}_{t}\right\|^{2}+\frac{\varsigma t}{P_{t}}\right)^{\lambda_{t}} P_{t}^{2\left(\lambda_{t}-\lambda_{\bar{t}}\right)}}
\end{aligned}
$$

which decouples the source beamforming matrix $\mathbf{B}_{t}$. Denoting that $\frac{\left(\left\|\mathbf{H}_{t}\right\|^{2}\right)^{\lambda_{\bar{t}}}}{\left(\left\|\tilde{\chi}_{t}\right\|^{2}+\frac{5 t}{P_{t}}\right)^{\lambda_{t}}}$ and $\widehat{\gamma_{t}}=\gamma_{t} P_{t}^{2\left(\lambda_{t}-\lambda_{\bar{t}}\right)}$, for the objective problem $T_{2}$, the SRINR constraint can be equivalently expressed as $\widehat{q}_{t}\left(\mathbf{B}_{t}\right) \geq \widehat{\gamma_{t}}$. It is clear that, after decoupling the source beamdforming $\mathbf{B}_{t}$ in the objective problem $T_{2}, \mathbf{B}_{t}$ only exists at the relay power constraint (4). By considering $\widehat{\mathbf{B}}_{t}=\operatorname{vec}\left(\widetilde{\mathbf{B}}_{t}\right) \operatorname{vec}\left(\widetilde{\mathbf{B}}_{t}\right)^{\dagger}$ with $\operatorname{vec}\left(\widetilde{\mathbf{B}}_{t}\right)=$ $\left[\operatorname{vec}\left(\mathbf{B}_{t}\right)^{T} \mathbf{0}_{1 \times M(N-M)}\right]^{T}$, similar to Appendix 2 in [8], we have the following linear matrix inequality (LMI) of the individual relay power constraints:

$$
\left[\begin{array}{ccc}
\widehat{\mathbf{B}}_{1}+\lambda_{1} \mathbf{I} & \widehat{\mathbf{B}}_{1} \operatorname{vec}\left(\widetilde{\mathbf{Q}}_{1}\right) & \mathbf{0} \\
\operatorname{vec}\left(\widetilde{\mathbf{Q}}_{1}\right)^{\dagger} \widehat{\mathbf{B}}_{1} & \aleph_{t} & \operatorname{vec}\left(\widetilde{\mathbf{Q}}_{2}\right)^{\dagger} \widehat{\mathbf{B}}_{2} \\
\mathbf{0} & \widehat{\mathbf{B}}_{2} \operatorname{vec}\left(\widetilde{\mathbf{Q}}_{2}\right) & \widehat{\mathbf{B}}_{2}+\lambda_{2} \mathbf{I}
\end{array}\right] \succeq 0,
$$

where $\aleph_{t}=\sum_{t=1}^{2} \operatorname{vec}\left(\widetilde{\mathbf{F}}_{i, t}\right)^{\dagger} \widehat{\mathbf{B}}_{t} \operatorname{vec}\left(\widetilde{\mathbf{F}}_{i, t}\right)-\lambda_{1} \omega_{i} \alpha_{i}^{2}-$ $\lambda_{2} \omega_{i} \beta_{i}^{2}-\widehat{P}_{R_{i}}$ and $\widetilde{\mathbf{Q}}_{t}=\mathbf{W}_{i} \widetilde{\mathbf{F}}_{i, t}$. Based on which, the optimization problem $T_{2}$ can be approximately converted into the following version as

$$
\begin{aligned}
T_{3}: \max _{\mathbf{B}_{t}, \mathbf{w}_{i}} & \prod_{t=1}^{2} \widehat{\gamma_{t}} \text { for } t=1,2, \\
\text { s.t. } & \widehat{q}_{t}\left(\mathbf{B}_{t}\right) \geq \widehat{\gamma_{t}},(21),\left\|\mathbf{B}_{t}\right\|^{2} \leq P_{t} .
\end{aligned}
$$

It is easy to see that the problem $T_{3}$ is still hard to obtain the optimal solution straightforwardly, therefore, we try to convert it into LMI version. It is clear that the denominator of $\widehat{q}_{t}\left(\mathbf{B}_{t}\right)$ can be approximately reexpressed as

$$
\tau_{t}+\frac{\varsigma_{t}}{P_{t}}
$$

where $\tau_{t}$ is obtained from

$$
\begin{aligned}
\left\|\widetilde{\chi}_{\bar{t}}\right\|^{2} & \leq\left\|\sum_{i=1}^{L} \widetilde{\mathbf{G}}_{\bar{t}, i} \mathbf{W}_{i}^{\sharp} \Delta_{\mathbf{F}_{i, \bar{t}}}\right\|^{2}+\left\|\sum_{i=1}^{L} \Delta_{\mathbf{G}_{\bar{t}, i}} \mathbf{W}_{i}^{\sharp} \widetilde{\mathbf{F}}_{i, \bar{t}}\right\|^{2} \\
& \leq 2 \sum_{i=1}^{L} \varrho_{t, i}^{2} \omega_{i}\left\|\widetilde{\mathbf{G}}_{\bar{t}, i}\right\|^{2}=\tau_{t} .
\end{aligned}
$$


Thus, letting $\varepsilon_{t}=\sqrt[\lambda_{t}]{\widehat{\gamma}_{t}\left(\tau_{t}+\frac{\varsigma t}{P_{t}}\right)^{\lambda_{t}}}$, the SRINR constraint becomes $\left\|\mathbf{H}_{t}\right\|^{2} \geq \varepsilon_{t}$ which has the LMI form as follows:

$$
\left[\begin{array}{cc}
-\varepsilon_{t} & -\mathbf{H}_{t} \\
\mathbf{H}_{t}^{\dagger} & \mathbf{I}_{M}
\end{array}\right] \succeq 0
$$

Since

$$
\mathbf{H}_{t}=\Lambda_{\mathbf{G}, \mathbf{F}}+\sum_{i=1}^{L} \mathbf{M}_{\mathbf{G}_{\bar{t}, i}} \operatorname{vec}\left(\Delta_{\mathbf{G}_{\bar{t}, i}}\right)+\sum_{i=1}^{L} \mathbf{M}_{\mathbf{F}_{i, t}} \operatorname{vec}\left(\Delta_{\mathbf{F}_{i, t}}\right) \text {, }
$$

with

$$
\begin{aligned}
\mathbf{M}_{\mathbf{G}_{\bar{t}, i}} & =\sum_{i=1}^{L}\left(\mathbf{W}_{i}^{\sharp} \widetilde{\mathbf{F}}_{i, t}\right)^{T} \otimes \mathbf{I}, \\
\Lambda_{\mathbf{G}, \mathbf{F}} & =\sum_{i=1}^{L} \operatorname{vec}\left(\widetilde{\mathbf{G}}_{\bar{t}, i} \mathbf{W}_{i} \widetilde{\mathbf{F}}_{i, t}\right)
\end{aligned}
$$

and

$$
\mathbf{M}_{\mathbf{F}_{i, t}}=\sum_{i=1}^{L} \mathbf{I} \otimes\left(\widetilde{\mathbf{G}}_{\bar{t}, i} \mathbf{W}_{i}\right),
$$

substituting (25) back into (24) and using the S-Lemma [35], the LMI version of the SRINR constraint can be finally denoted as

$$
\left[\begin{array}{cccc}
\Theta_{t}-\sum_{j=1}^{2 l} \phi_{j} \mathbf{q}_{2}^{\dagger} \mathbf{q}_{2} & \xi_{1, t} \boldsymbol{\Xi}_{1} & \ldots & \xi_{2 l, t} \boldsymbol{\Xi}_{2 l} \\
-\xi_{1, t} \boldsymbol{\Xi}_{1}^{\dagger} & \phi_{1} \mathbf{I}_{M N} & \cdots & \mathbf{0} \\
\vdots & \vdots & \ddots & \vdots \\
-\xi_{2 l, t} \boldsymbol{\Xi}_{2 l}^{\dagger} & \mathbf{0} & \cdots & \phi_{2 l} \mathbf{I}_{M N}
\end{array}\right] \succeq 0
$$

where $\mathbf{q}_{2}=\left[-1, \mathbf{0}_{1 \times M^{2}}\right]$ and $\phi_{j} \geq 0$ are the slack variables for $j=1, \ldots, 2 l . \mathbf{\Xi}_{i}=\left[\mathbf{0}_{M^{2} \times 1}, \mathbf{M}_{\mathbf{G}_{\bar{t}, i}^{H}}^{H}\right]$, and $\xi_{i, t}=\left\|\Delta_{\mathbf{G}_{\bar{t}, i}}\right\|$ for $i=1, \ldots, l$, as well as $\mathbf{\Xi}_{i}=\left[\mathbf{0}_{M^{2} \times 1}, \mathbf{M}_{\mathbf{F}_{i, t}}^{H}\right]$, and $\xi_{i, t}=$ $\left\|\Delta_{\mathbf{F}_{i, t}}\right\|$ for $i=l+1, \ldots, 2 l$, with

$$
\Theta_{t}=\left[\begin{array}{cc}
-\varepsilon_{t} & -\Lambda_{\mathbf{G}, \mathbf{F}} \\
\Lambda_{\mathbf{G}, \mathbf{F}}^{\dagger} & \mathbf{I}_{M^{2}}
\end{array}\right] .
$$

Thus, optimization problem $T_{3}$ is recast as

$$
\begin{array}{ll}
T_{4}: & \max _{\mathbf{B}_{t}, \mathbf{W}_{i}} \prod_{t=1}^{2} \widehat{\gamma}_{t} \\
\text { s.t. } & (21),(29),\left\|\mathbf{B}_{t}\right\|^{2} \leq P_{t},\left\|\mathbf{W}_{i}\right\|^{2} \leq \omega_{i}, \tau_{t} \geq 0, \varsigma_{t} \geq 0, \\
\lambda_{t} \geq 0, v_{i} \geq 0, \phi_{j} \geq 0, \forall t=1,2, \forall i=1, \ldots, l, \forall j=1, \ldots, 2 l .
\end{array}
$$

Since the objective problem $T_{3}$ is a biconvex semidefinite program (SDP) which can be efficiently solved by the following alternating algorithm by using CVX [34]:

1. Initialize: $\xi, N_{\max }, \mathbf{B}_{t}^{(n)}$ and $\mathbf{W}_{i}^{(n)}$, set $n=0$;

2. Repeat: For $n=0$ to $N_{\max }$
1: with fixed $\mathbf{B}_{t}^{(n-1)}$ update $\mathbf{W}_{i}^{(n)}$ by solving $T_{4}$;

2: for given $\mathbf{W}_{i}^{(n)}$ update $\mathbf{B}_{t}^{(n)}$ by solving $T_{4}$;

3: If $\prod_{t=1}^{2} \widehat{\gamma}_{t}^{(n)}-\prod_{t=1}^{2} \widehat{\gamma}_{t}^{(n-1)} \leq \xi$, break;

By initializing the small $\xi$ and setting the limitation of the number of iterations $N_{\max }$, the suboptimal solution can be obtained when $\prod_{t=1}^{2} \widehat{\gamma}_{t}^{(n)}-\prod_{t=1}^{2} \widehat{\gamma}_{t}^{(n-1)} \leq \xi$.

\section{Numerical results}

In this section, we examine the performance of the proposed decoupled scheme in terms of the average weighted SR and the convergent performance compared with the case with perfect CSI, the maximal-ratio transmission (MRT) and the SI-SRINR scheme in [8]. In all simulations, spatially uncorrelated Rayleigh fading channels with unit variance are assumed. The number of relay nodes is set to be $L=3$ with $M=N=2$, and the noise variance $\sigma_{R_{i}}^{2}$ and $\sigma_{S_{t}}^{2}$ are equally given as $\sigma^{2}=1$. We further set up that, in the proposed alternating algorithm, $N_{\max }=400$, $\xi=10^{-3}$, and the relay beamforming matrices are initialed at random, respectively. In the following figures, we use "Optimal," "Proposed perfect," "Proposed $\alpha_{i} / \beta_{i}$," and "SNR" to denote the performance upper bound, proposed scheme with perfect SCI, proposed scheme with NBEs of $\alpha_{i} / \beta_{i}$, and transmit SNR, respectively. Moreover, the performance upper bound "Optimal" is obtained by using the exhaustive search as a benchmark.

Figure 2 depicts the weighted SR performance versus transmit SNR in two considered cases: (1) $\lambda_{1}=6, \lambda_{2}=$ 1 ; $(2) \lambda_{1}=3, \lambda_{2}=1$, which compares to the optimal, perfect CSI and MRT schemes, respectively, with $\alpha_{i}=\beta_{i}=0.01$ and $\alpha_{i}=\beta_{i}=0.1$. In addition, it is worth noting that the perfect one is obtained with the NBE as $\alpha_{i}=\beta_{i}=0$ while the robust solutions are obtained by using the proposed alternating algorithm. It is clear that our proposed scheme has a relative advantage over the MRT maximization scheme and close to the optimal and perfect ones, especially for the smaller NBE case. In addition, the performance gain grows as the ratio between $\lambda_{1}$ and $\lambda_{2}$ increases. This is reasonable, since considering the high transmit SNR, (31) is very close to the original one which has the the negligible performance lose.

Figure 3 compares the convergence performance of the weighted SR for the proposed method with the SI-SRINR in [8], where we have fixed $\alpha_{i}^{2}=\beta_{i}^{2}=0.01$. Two cases are considered: (1) $\lambda_{1}=6, \lambda_{2}=1$ with SNR $=30 \mathrm{~dB}$ and (2) $\lambda_{1}=3, \lambda_{2}=1$ with SNR $=20 \mathrm{~dB}$. Results reveal that our proposal has an obvious satisfactory convergence over the SI-SINR scheme. This is because, for our proposed method, the weighted SR problem is approximately converted into a source beamforming decoupled one which significantly reduces the computational complexity. 


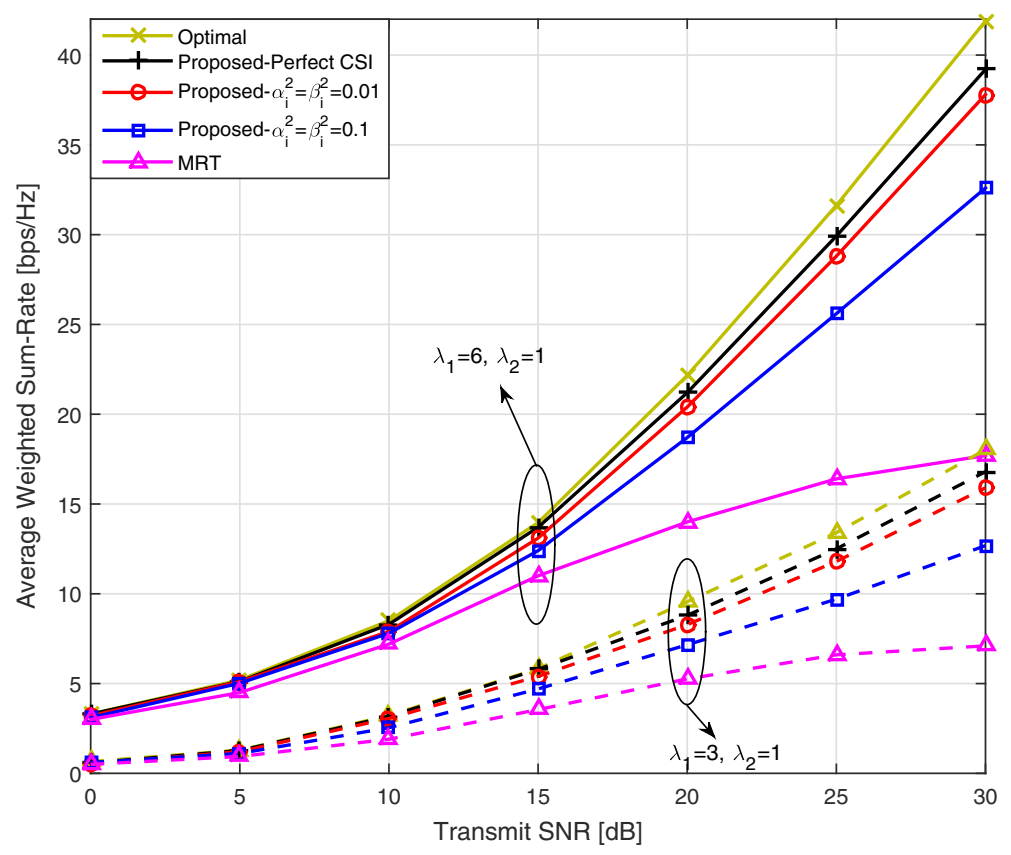

Fig. 2 The average weighted SR versus the transmit SNR

Specifically, by employing the Cauchy-Schwarz inequality and S-lemma, the optimal relay beamforming $\mathbf{W}_{i}$ is efficiently relaxed in $q_{t}\left(\mathbf{B}_{t}\right)$ which also simplifies the corresponding algorithm. In addition, it is observed that, for the higher transmit SNR, the convergence rate of our proposed scheme overwhelms the SI-SRINR one and both these two schemes provide the optimal solution with more iterations.

Figure 4 exhibits the average SR performance for the proposed method with the optimal scheme versus the transmission number $L$ with fixed $\alpha_{i}=\beta_{i}=0.01$ and $\lambda_{1}=\lambda_{2}=1$. Two cases are considered: (1) SNR $=30$

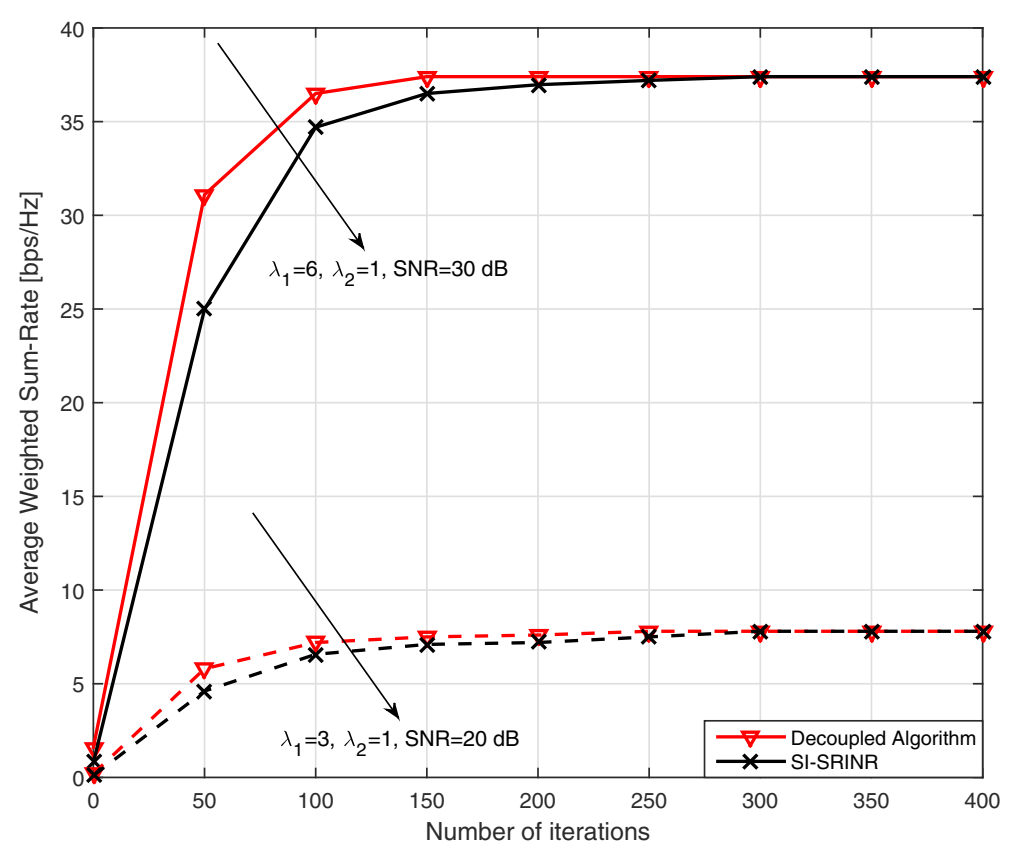

Fig. 3 The average weighted SR versus the number of iterations 


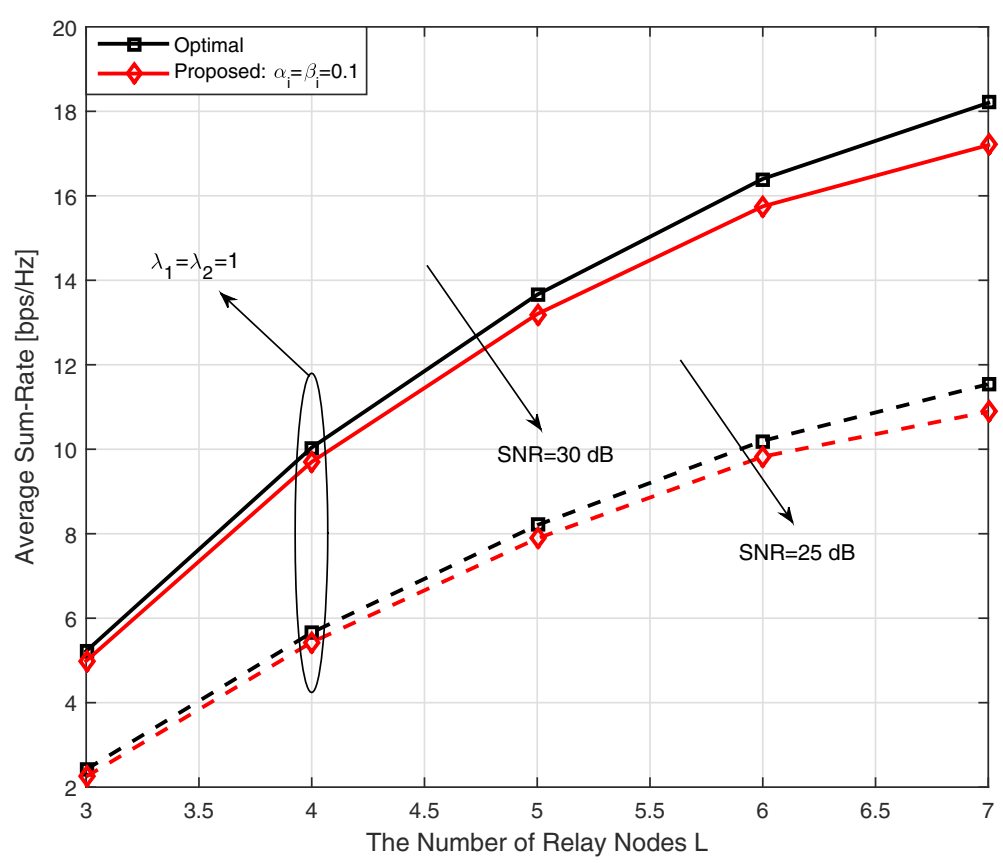

Fig. 4 The average weighted SR versus the number of relay $L$

$\mathrm{dB}$ and (2) $\mathrm{SNR}=25 \mathrm{~dB}$. It is clear that, from Fig. 3, it is easy to see that the performances of our proposed scheme have a negligible performance loss compared to the optimal scheme, especially for the smaller number of the relay, which supports the practical utility of our design. Remarkably, the performance gap between our proposed scheme and the optimal one is smaller for a lower SNR case.
Denoting $d_{1, i}$ and $d_{2, i}$ as the distance between $\left\{\mathrm{UE}_{1}, \mathrm{UE}_{2}\right\}$ and the relay $R_{i}$, respectively, the average transmit SNRs, which take into account the relay positions, can be written as

$$
\zeta_{1, i}^{*}=\frac{\bar{\zeta}_{1, i}}{d_{1, i}^{\delta}}, \zeta_{2, i}^{*}=\frac{\bar{\zeta}_{2, i}}{d_{2, i}^{\delta}}
$$

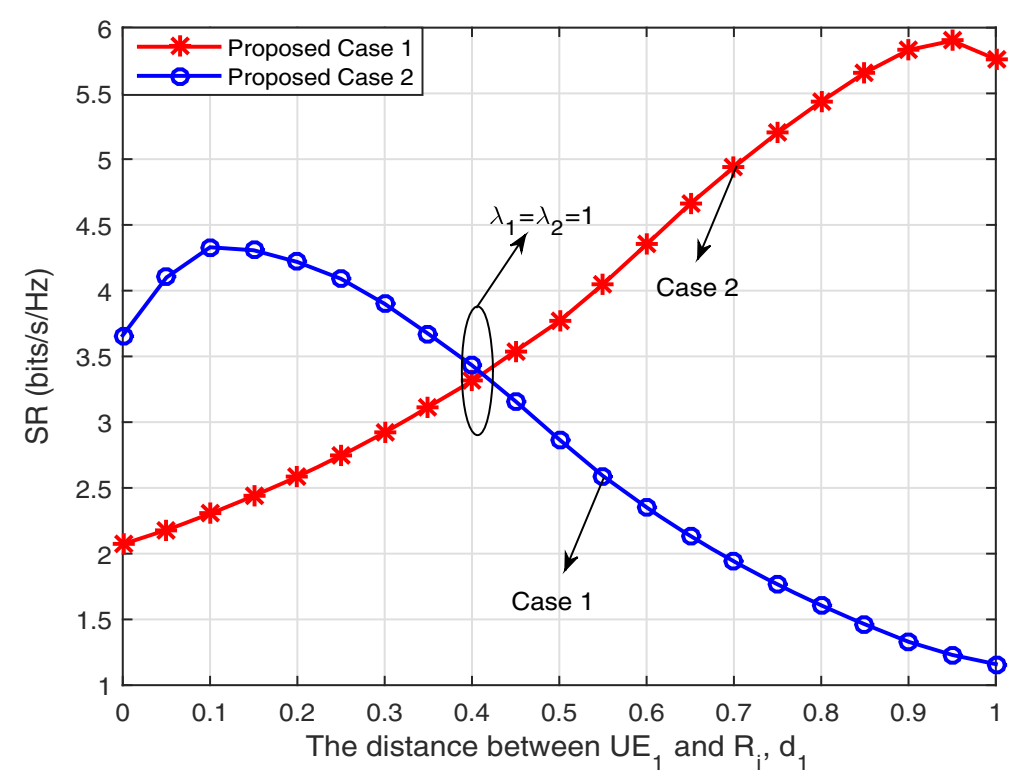

Fig. 5 The achievable weighted SR versus the distance between $U E_{t}$ and $R_{i}$ 
where $\bar{\zeta}_{1, i}=\frac{P_{1}}{\sigma_{R_{i}}^{2}}, \bar{\zeta}_{2, i}=\frac{P_{2}}{\sigma_{R_{i}}^{2}}$ and $\delta$ is the path loss exponent. Figure 5 depicts the maximum weighted SR of our proposed decoupled beamforming design for different values of $d_{1, i}$ with fixed $\lambda_{1}=\lambda_{2}=1, \delta=3$ in two considered system setups: (1) case $1: \bar{\zeta}_{1}=20 \mathrm{~dB}, \bar{\zeta}_{2}=15 \mathrm{~dB}$; (2) case $2: \bar{\zeta}_{1}=20 \mathrm{~dB}, \bar{\zeta}_{2}=25 \mathrm{~dB}$. For simplicity, we further assume that $d_{1,1}=d_{1,2}=d_{1,3}=d_{1}$ and $d_{2,1}=d_{2,2}=d_{2,3}=d_{2}$ with $d_{1}+d_{2}=1$. One can see that the achieved SR for case 2 shows a higher performance than case 1 due to a lager source power considered for $\mathrm{UE}_{2}$. Moreover, the optimal relay positions occur when $d_{1}$ is close to 0.1 for case 1 and $d_{1} \rightarrow 0.95$ for case 2 . It is reasonable since that the power at $\mathrm{UE}_{1}$ is greater than the power at $\mathrm{UE}_{2}$ for case 1 , while an opposite consideration is performed in case 2 .

\section{Conclusions}

In this paper, we investigated the decoupled beamforming techniques in the distributed two-way multi-relay networks with imperfect and reciprocal CSI. In order to maximize the weighted SR, the objective problem is first converted into a decoupled one by employing the CauchySchwarz inequality and S-lemma, and then, the optimal relay beamforming design is also investigated with the LMI versions of the semi-infinite constraints. Based on which, the objective problem can be efficiently solved by the proposed alternating algorithm. By means of the numerical results, it has been shown that our proposed scheme not only has the advantage in the term of the SR but also is with the satisfactory convergence compared to the existing works.

\section{Acknowledgements \\ The authors would gratefully acknowledge the grants from the National Natural Science Foundation of China (61371113 and 61401241), Nantong University-Nantong Joint Research Center for Intelligent Information Technology (KFKT2016B04 and KFKT2017B02), the Science and Technology Program of Nantong (GY22017013), and the Brain Korea BK21 plus. Finally, we would like to thank the Editor for your constructive remarks and careful reading of our paper, which were essential in improving the overall presentation of the paper.}

\section{Authors' contributions}

WD, JC, and GZ conceived and designed the study. WD and XZ performed the simulations. WD and GZ wrote the paper. $L, W W, J C$, and GZ reviewed and edited the manuscript. All authors read and approved the final manuscript.

\section{Competing interests}

The authors declare that they have no competing interests.

\section{Publisher's Note}

Springer Nature remains neutral with regard to jurisdictional claims in published maps and institutional affiliations.

\footnotetext{
Author details

${ }^{1}$ School of Electronics and Information, Nantong University, Nantong, China. ${ }^{2}$ Division of Electronic and Information Engineering, Chonbuk National University, Chonju, Korea. ${ }^{3}$ Department of ECE, CAllT, Chonbuk National University, Chonju, Korea. ${ }^{4}$ Nantong Research Institute for Advanced Communication Technologies, Nantong 226019, Jiangsu, China.
}

Received: 3 August 2017 Accepted: 24 April 2018

Published online: 18 May 2018

\section{References}

1. SHan, C Yang, M Bengtsson, User scheduling for cooperative base station transmission exploiting channel asymmetry. IEEE Trans. Commun. 61(4), 1426-1435 (2013)

2. H Lin, F Gao, S Jin, G Ye Li, A new view of multi-user hybrid massive MIMO: non-orthogonal angle division multiple access. IEEE J. Sel. Areas Commun. 35(10), 2268-2280 (2017)

3. H Xie, F Gao, S Jin. An overview of low-rank channel estimation for massive MIMO systems.IEEE Access. 4(99), 7313-7321 (2016)

4. H Xie, F Gao, S Zhang, S Jin, A unified transmission strategy for TDD/FDD massive MIMO systems with spatial basis expansion model. IEEE Trans. Veh. Technol. 66(4), 3170-3184 (2017)

5. J Joung, J Choi, Linear precoder design for an AF two-way MIMO relay node with no source node precoding. IEEE Trans. Veh. Technol. 66(11), 10526-10531 (2017). to appear

6. R Budhiraja, B Ramamurthi, Transceiver design for nonconcurrent two-way MIMO AF relaying with QoS guarantees. IEEE Trans. Veh. Technol. 65(12), 9651-9661 (2016)

7. Y Dai, X Dong, Power allocation for multi-pair massive MIMO two-way AF relaying with linear processing. IEEE Trans. Wireless Commun. 15(9), 5932-5946 (2016)

8. W Duan, M Wen, X Jiang, Y Yan, M Lee, Sum-rate maximization and robust beamforming design for MIMO two-way relay networks with reciprocal and imperfect CSI. EURASIP J. Commun. and Network. (2016). Atical ID: 157

9. Q Cui, TYuan, X Tao, AA Dowhuszko, R Jantti, Energy efficiency analysis of two-way DF relay system with non-ideal power amplifiers. IEEE Commun. Lett. 18(7), 1254-1257 (2014)

10. J Gao, SA Vorobyov, H Jiang, J Zhang, M Haardt, Sum-rate maximization with minimum power consumption for MIMO DF two-way relaying — part II: network optimization. IEEE Trans. Signal Process. 61(14), 3578-3591 (2013)

11. Z Zhao, M Peng, Z Ding, W Wang, H Chen, Denoise-and-forward network coding for two-way relay MIMO systems. IEEE Trans. Veh. Technol. 63(2), 775-788 (2014)

12. M Masjedi, A Hoseini, S Gazor, Non-coherent detection and denoise-and-forward two-way relay networks. IEEE Trans. Commun 64(11), 4497-4505 (2016)

13. CWang, $\mathrm{H}$ Chen, Q Yin, A Feng, AF Molisch, Multi-user two-way relay networks with distributed beamforming. IEEE Trans. Wireless Commun. 10(10), 3460-3471 (2011)

14. V Nassab, S Shahbazpanahi, A Grami, Optimal distributed beamforming for two-way relay networks. IEEE Trans. Signal Process. 58(3), 1238-1250 (2010)

15. Y Rong, Joint source and relay optimization for two-way linear nonregenerative $\mathrm{MIMO}$ relay communications. IEEE Trans. Signal Process. 60(12), 6533-6546 (2012)

16. W Cheng, M Ghogho, Q Huang, D Ma, J Wei, Maximizing the sum-rate of amplify-and-forward two-way relaying networks. IEEE Commun. Lett. 18(11), 635-638 (2011)

17. J-M Kang, H-M Kim, Training designs for estimation of spatially correlated fading channels in MIMO amplify-and-forward two-way multi-relay networks. IEEE Commun. Lett. 20(4), 772-775 (2016)

18. J-M Kang, I-M Kim, H-M Kim, Optimal training design for MIMO-OFDM two-way relay networks. IEEE Trans. Commun. 65(9), 3675-3690 (2017)

19. R Louie, Y Li, B Vucetic, Practical physical layer network coding for two-way relay channels: performance analysis and comparison. IEEE Trans. Wireless Commun. 9(2), 764-777 (2010)

20. F Jiang, C Zhu, J Peng, W Liu, Z Zhu, Y He, Joint relay and jammer selection and power control for physical layer security in two-way relay networks with imperfect CSI. Wireless Pers. Commun. 85, 841-862 (2015)

21. CWang, $X$ Dong, Y Shi, Robust relay design for two-way multi-antenna relay systems with imperfect CSI. J. Commun. and Networks. 16(1), 45-55 (2014)

22. F Jiang, C Zhu, J Peng, W Liu, Z Zhu, Y He, Multi-pair two-way massive MIMO AF full-duplex relaying with imperfect CSI over Ricean fading channels. IEEE Access. 4, 4933-4945 (2016)

23. S Salari, MZ Amirani, I Kim, D Kim, J Yang, Distributed beamforming in two-way relay networks with interference and imperfect CSI. IEEE Trans. Wireless Commun. 15(6), 4455-4469 (2016) 
24. P Ubaidulla, S Aissa, Robust two-way cognitive relaying: precoder designs under interference constraints and imperfect CSI. IEEE Trans. Wireless Commun. 13(5), 2478-2489 (2014)

25. C Song, H Park, H Lee, I Lee, Robust beamforming designs for nonregenerative multipair two-way relaying systems. IEEE Trans. Wireless Commun. 65(9), 7802-7808 (2016)

26. M Gastpar, M Vetterli, On the capacity of large Gaussian relay networks. IEEE Trans. Inf. Theory. 51(3), 765-779 (2005)

27. Y Huang, D Palomar, S Zhang, Lorentz-positive maps and quadratic matrix inequalities with applications to robust MISO transmit beamforming. IEEE Trans. Signal Process. 61(5), 1121-1130 (2013)

28. Y Rong, Joint source and relay optimization for two-way linear non-regenerative MIMO relay communications. IEEE Trans. Signal Process. 60(12), 6533-6546 (2003)

29. Q Zhang, Q Li, J Qin, Beamforming design for OSTBC-based AF-MIMO two-way relay networks with simultaneous wireless information and power transfer. IEEE Trans. Veh. Technol. 65(9), 7285-7296 (2016)

30. XXie, H Yang, AV Vasilakos, Robust transceiver design based on interference alignment for multi-user multi-cell MIMO networks with channel uncertainty. IEEE Access. 5, 5121-5134 (2017)

31. Y Tang, J Xiong, D Ma, X Zhang, Robust artificial noise aided transmit design for MISO wiretap channels with channel uncertainty. IEEE Commun. Lett. 17(1), 2096-2099 (2013)

32. P Ubaidulla, S Aissa, Robust two-way cognitive relaying: precoder designs under interference constraints and imperfect CSI. IEEE Trans. Wireless Commun. 13(5), 2478-2489 (2014)

33. Q Li, Q Zhang, J Qin, Robust beamforming for cognitive multi-antenna relay networks with bounded channel uncertainties. IEEE Trans. Commun. 62(2), 478-487 (2014)

34. S Boyd, L Vandenberghe, Convex optimization. (Cambridge University Press, Cambridge, 2004)

35. J Liu, F Gao, Z Qiu, Robust transceiver design for downlink multiuser MIMO AF relay systems. IEEE Trans. Wireless Commun. 14(4), 2218-2231 (2015)

\section{Submit your manuscript to a SpringerOpen ${ }^{\circ}$ journal and benefit from:}

- Convenient online submission

- Rigorous peer review

- Open access: articles freely available online

- High visibility within the field

- Retaining the copyright to your article

Submit your next manuscript at $>$ springeropen.com 